\title{
Abdomen agudo en el adulto mayor
}

\author{
Ricardo Espinoza G, Paulina Balbontín M, \\ Sebastián Feuerhake L, Cecilia Piñera M.
}

\section{Acute abdomen in the elderly}

Background: Nearly $10 \%$ of emergency consultations are due to acute abdominal pain. In people over 65 years old, it can have atypical presentations, that retard the correct diagnosis and worsens prognosis. Aim: To study the causes, evolution and prognosis of acute abdomen in the elderly. Material and methods: Prospective study of 45 patients aged more than 65 years old (mean age \pm SD, 75.7 $\pm 7.7,51 \%$ men) and 221 patients of less than 65 years old (mean age \pm SD, $36.7 \pm 14.0,48 \%$ men), consulting for acute abdomen in the emergency room. Results: Sixty six percent of elderly patients had concomitant diseases, that were multiple in $63 \%$. In this age group, the causes accounting for $71 \%$ of acute abdominal pain were bilio-pancreatic diseases (31.1\%), intestinal adhesive obstruction (17.7\%), complicated abdominal wall hernia (13.7\%), and complications of peptic ulcer disease (8.9\%). Sixty four percent required surgical treatment and, in almost 50\% the surgical risk was classified in ASA III or IV, according to the American Society of Anesthesiology. Thirty one percent had postoperative complications. Compared with their younger counterparts, elderly patients required significantly $(\mathrm{p}<0.05)$ more admissions to intensive care units ( 2.7 and $24.2 \%$ respectively), more connections to mechanical ventilation (1.4 and $8.9 \%$ respectively) and longer hospital stays (5.4 \pm 7.4 and $12.4 \pm 10.9$ days, respectively). In this series overall mortality was $6.7 \%$, being $0.6 \%$ for young patients and $11.1 \%$ for the surgical group over 65 years old. Conclusions: Acute abdomen in the elderly has a high rate of complications and mortality. According to the causes of acute abdomen in this group, evaluation in the emergency setting with an ultrasonography may be very useful. In the elderly, elective correction of potential causes of acute abdomen should be done (Rev Méd Chile 2004; 132: 1505-12).

(Key Words: Abdomen, acute; Aged; Surgical procedures, operative)

Recibido el 21 de noviembre, 2003. Aceptado en versión corregida el 8 de octubre, 2004.

Facultad de Medicina, Universidad de los Andes.

$\mathrm{C}$ omo abdomen agudo reconocemos una entidad clínica que, de acuerdo a la definición proporcionada por la National Library of Medicine, corresponde a un síndrome caracterizado por dolor abdominal que por su gravedad y los síntomas y signos asociados, que simulan una peritonitis aguda, puede corresponder a una pato-

Correspondencia a: Dr. Ricardo Espinoza G. Av. San Carlos de Apoquindo 2200. E mail: respinoza@uandes.cl logía quirúrgica, pero que no necesariamente lo es. Por este motivo, hay causas de abdomen agudo de tratamiento médico. El término se ha transformado en un diagnóstico operativo con que el médico de urgencia trabaja para llegar al diagnóstico más preciso, causante del síndrome, luego de la observación y aplicación de una variada gama de exámenes de laboratorio y de imágenes. En muchas oportunidades, sin embargo, la forma de establecer el diagnóstico definitivo, es una laparotomía de urgencia. 
Se estima que hasta $10 \%$ de las consultas médicas de urgencia son motivadas por un cuadro de abdomen agudo (AA) y, como se ha expresado, su correcta evaluación es el paso fundamental para instituir un tratamiento adecuado y oportuno ${ }^{1}$. Por cierto que ello es especialmente válido para las condiciones que van a requerir una resolución quirúrgica.

Se ha observado que la población mayor de 65 años requiere cada vez con mayor frecuencia procedimientos quirúrgicos ${ }^{1,2}$ y por otro lado, se sabe que en ellos las presentaciones clínicas de las diferentes entidades responsables de un abdomen agudo son atípicas, siendo muchas veces el deterioro agudo funcional o cognitivo el primer signo de un cuadro de este tipo ${ }^{1-3}$. Ello explica, en parte, que las cifras de morbilidad y mortalidad por causas específicas de urgencia, sean más elevadas en la población mayor ${ }^{4-7}$. Además, hay que agregar la coexistencia de condiciones médicas de diversa gravedad que reducen sus reservas fisiológicas y facilitan la aparición de complicaciones 3,8 .

En nuestro país la población mayor de 65 años ha aumentado considerablemente ${ }^{9}$, sobrepasando el $10 \%$ en el año 2000 y no conocemos estudios locales que hayan investigado el tema del AA en este particular grupo etario, en cuanto a sus causas, el tratamiento y sus resultados.

Nuestro objetivo fue conocer las principales causas de abdomen agudo que motivaron la hospitalización de una población mayor de 65 años y comparar evolución, morbilidad y mortalidad con un grupo de menor edad.

\section{PACIENTES Y MÉTOdO}

Se efectuó un estudio prospectivo, con seguimiento intrahospitalario, de todos los pacientes adultos que ingresaran a través del Servicio de Urgencia por un cuadro catalogado como abdomen agudo. Para el análisis de las causas se consideró el diagnóstico definitivo al momento del alta hospitalaria o del fallecimiento. Se consideró como patología bilio-pancreática a la patología litiásica aguda, excluyéndose los cánceres de vesícula y vía biliar. La patología neoplásica abdominal que se presentó como un abdomen agudo, se agrupó como tumor digestivo y correspondió a tumores de vesícula biliar, páncreas y colon, con carcinomatosis peritoneal. Se agruparon como obstrucción intestinal los casos producidos por bridas.

Este estudio se realizó en el Hospital Parroquial de San Bernardo, entre julio y diciembre del año 2002. El diseño incluyó el registro de variables demográficas como sexo y edad y la presencia de patología asociada. Se registró el número de consultas previas por el mismo cuadro y la proporción de pacientes que requirió tratamiento quirúrgico. En ese caso, se estimó el riesgo anestésico de acuerdo a la definición de la Sociedad Americana de Anestesiólogos (American Society of Anesthesiologists, ASA) ${ }^{10}$.

Se evaluó la necesidad de atención en Unidad de Cuidados Intensivos, utilización de ventilación mecánica, días totales de hospitalización, morbilidad y mortalidad. Para el análisis se comparó el subgrupo de pacientes mayores de 65 años con los menores de dicha edad.

Para fines estadísticos se aplicó el test de chi cuadrado, considerándose significativo un valor de $\mathrm{p}<0,05$.

\section{RESULTADOS}

Durante el período señalado 30.089 pacientes adultos consultaron en el Servicio de Urgencia del Hospital Parroquial de San Bernardo. De ese total fueron ingresados 1.442 (4,8\%), y de ellos, 266 por un cuadro de abdomen agudo (18,4\%). De éstos últimos, 221 eran de hasta 64 años de edad (Grupo A) y los 45 restantes tenían 65 o más años (Grupo B). En el primero, la edad promedio fue de $36,7 \pm 14,0$ años y se elevó a $75,7 \pm 7,7$ en el segundo. En ambos, la proporción por sexo fue similar: para el grupo A hubo 105 pacientes de sexo masculino y 116 de sexo femenino, siendo en el B 23 y 22, respectivamente.

En el Grupo A, 70,6\% de los pacientes no refería antecedentes mórbidos, existiendo patología preexistente en $66,7 \%$ del otro grupo (p $<0,05$ ). Cuando existía patología previa, ésta era múltiple en $30,8 \%$ de los casos del grupo A; a diferencia de $63,3 \%$ del grupo B. Las principales patologías asociadas se pueden observar en la Tabla 1, destacando la significativa mayor inciden- 
cia de hipertensión arterial, diabetes mellitus y enfermedades neoplásicas, en la población mayor de 65 años.

Los diagnósticos definitivos de los pacientes de cada grupo aparecen en la Tabla 2. Destaca que en el Grupo A, casi la mitad de los pacientes presentó un cuadro apendicular agudo, patología que fue la causa del AA en sólo 6,7\% de los pacientes de mayor edad. En ambos grupos la patología bilio-pancreática fue frecuente. En el grupo B fue significativamente más frecuente la patología derivada de una hernia complicada y los cuadros de obstrucción intestinal. Así, sobre 70\% de los diagnósticos en la población menor de 65 años estuvo dado sólo por dos patologías: apendicular y bilio-pancreática. En el Grupo B, 71\% de las causas de abdomen agudo estuvo dado por patología bilio-pancreática, obstrucción intestinal, hernia complicada y complicaciones de una úlcera péptica.

Para cada grupo, en una proporción similar, el diagnóstico de egreso fue diferente al inicial, $18,1 \%$ para Grupo A y $15,6 \%$ para B (p: NS). Tampoco observamos diferencias entre ambos grupos al comparar número de consultas previas al ingreso, puesto que por la misma causa, y en

Tabla 1. Patología asociada en $\mathbf{2 6 6}$ pacientes adultos con abdomen agudo

\begin{tabular}{|lrrrrc|}
\hline Tipo & \multicolumn{2}{c}{ Grupo A } & \multicolumn{2}{c|}{ Grupo B } & \\
& $\mathrm{n}$ & $\%$ & $\mathrm{n}$ & $\%$ & \\
\hline Hipertensión arterial & 25 & 11,3 & 21 & 46,7 & $\mathrm{p}<0,05$ \\
Obesidad & 13 & 5,9 & 0 & - & $\mathrm{p} \mathrm{NS}$ \\
Diabetes Mellitus & 12 & 5,4 & 9 & 20,0 & $\mathrm{p}<0,05$ \\
Alcoholismo & 9 & 4,1 & 1 & 2,2 & $\mathrm{p} \mathrm{NS}$ \\
Enfermedades respiratorias & 4 & 1,8 & 2 & 2,2 & $\mathrm{p} \mathrm{NS}$ \\
Enfermedades cardiovasculares & 4 & 1,8 & 6 & 13,3 & $\mathrm{p}<0,05$ \\
Tabaquismo & 4 & 1,8 & 1 & 2,2 & $\mathrm{p} \mathrm{NS}$ \\
Insuficiencia renal crónica & 1 & 0,5 & 3 & 6,7 & $\mathrm{p}<0,05$ \\
Enfermedad neoplásica & 1 & 0,5 & 7 & 15,6 & $\mathrm{p}<0,05$ \\
Otros & 9 & 4,1 & 1 & 2,2 & $\mathrm{p} \mathrm{NS}$ \\
\hline
\end{tabular}

Tabla 2. D iagnóstico definitivo en $\mathbf{2 6 6}$ pacientes adultos con abdomen agudo

\begin{tabular}{|lrrrrc|}
\hline Patología & \multicolumn{2}{c}{ Grupo A } & \multicolumn{2}{c|}{ Grupo B } \\
& $\mathrm{n}$ & $\%$ & $\mathrm{n}$ & $\%$ & \\
\hline Apendicular & 106 & 47,9 & 3 & 6,7 & $\mathrm{p}<0,05$ \\
Bilio-pancreático & 52 & 23,5 & 14 & 31,1 & $\mathrm{p} \mathrm{NS}$ \\
Dolor abdominal no específico & 16 & 7,2 & 1 & 2,2 & $\mathrm{p} \mathrm{NS}$ \\
Hernia complicada & 11 & 4,9 & 6 & 13,3 & $\mathrm{p}<0,05$ \\
Patología ginecológica & 7 & 3,2 & 1 & 2,2 & $\mathrm{p} \mathrm{NS}$ \\
Adenitis mesentérica & 5 & 2,3 & 0 & - & $\mathrm{p} \mathrm{NS}$ \\
Obstrucción intestinal & 4 & 1,8 & 8 & 17,7 & $\mathrm{p}<0,05$ \\
Enfermedad ulcerosa péptica & 4 & 1,8 & 4 & 8,9 & $\mathrm{p}<0,05$ \\
Enfermedad diverticular colon & 3 & 1,4 & 2 & 4,4 & $\mathrm{p} \mathrm{NS}$ \\
Tumor digestivo & 3 & 1,4 & 3 & 6,7 & $\mathrm{p}<0,05$ \\
Infección tracto urinario & 2 & 0,9 & 1 & 2,2 & $\mathrm{p} \mathrm{NS}$ \\
Accidente vascular mesentérico & 1 & 0,5 & 1 & 2,2 & $\mathrm{p}$ NS \\
Otros (incluye Meckel) & 7 & 3,2 & 1 & 2,2 & $\mathrm{p}$ NS \\
\hline
\end{tabular}


los tres días anteriores, hubo otra consulta en $42,5 \%$ de los pacientes del grupo A y en $42,2 \%$ de los del B.

El tratamiento fue quirúrgico en 181 pacientes del grupo A (81,9\%) y en $64,4 \%$ de aquellos del grupo B, (29 de los 45). Para el primer grupo hubo 2,3\% de laparotomías en blanco y ninguna en el grupo de mayor edad. En toda la serie, 10 pacientes se resolvieron exclusivamente con cirugía endoscópica, en proporción similar para ambos grupos. Para los casos sometidos a cirugía abierta convencional, 173 pacientes en grupo A y 27 en el B, la clasificación ASA se observa en la Tabla 3. $\mathrm{Si}$ bien todas las cirugías fueron de urgencia, 77,5\% de los pacientes menores de 65 años catalogó como ASA I, y 17,3\% como ASA II, a diferencia de lo observado en el grupo $B$, en que sólo $3,7 \%$ era ASA I y casi $50 \%$ ASA III o IV.

Se observaron una o más complicaciones en $12,2 \%$ de los pacientes del grupo A y en $31,1 \%$ de los del B, siendo múltiple en $22,2 \%$ del primer grupo y en $71,4 \%$ de los de mayor edad. Entre los operados, y para ambos grupos, fue frecuente la infección de herida operatoria. Para el grupo B, fue significativamente más frecuente la infección extra-abdominal (respiratoria y urinaria), los cuadros de sepsis e insuficiencia renal postoperatoria. Complicaciones cardiovasculares (arritmias y síndromes coronarios agudos) y cuadros de trombosis venosa y embolia pulmonar sólo se observaron entre los de más de 65 años (Tabla 4).

Sólo seis pacientes (2,7\%) menores de 65 años (grupo A), requirieron hospitalización en Unidad de Cuidados Intensivos, cifra que se elevó a 24,4\% de los del grupo B $(p<0,05)$. En este último grupo también fue significativamente más frecuente la utilización de ventilación mecánica (Tabla 5).

La mortalidad global de la serie fue de cuatro pacientes. Falleció uno de los 221 del grupo A $(0,5 \%)$ y tres entre los 45 del B $(6,7 \%)$. Si analizamos sólo aquellos casos operados, la mortalidad fue de 0,6\% y 11,1\% para cada grupo, respectivamente. Para ambos casos, la diferencia fue significativa estadísticamente. Del grupo A, falleció un paciente de 48 años, ASA I, que ingresó tardíamente por una perforación rectal. Los fallecidos del B corresponden a un paciente de 80 años, ASA III con una obstrucción intestinal;

Tabla 3. Tratamiento quirúrgico y riesgo anestésico (ASA) en 266 pacientes adultos con abdomen agudo

\begin{tabular}{|c|c|c|c|c|c|}
\hline & \multicolumn{2}{|c|}{ Grupo A } & \multicolumn{2}{|c|}{ Grupo B } & \\
\hline & $\mathrm{n}$ & $\%$ & $\mathrm{n}$ & $\%$ & \\
\hline Quirúrgico por laparotomía* & 173 & 78,3 & 27 & 60,0 & \\
\hline Quirúrgico endoscópico & 8 & 3,6 & 2 & 4,4 & \\
\hline ASA* & 134 & 77,5 & 1 & 3,7 & $p<0,05$ \\
\hline II & 30 & 17,3 & 13 & 48,1 & $p<0,05$ \\
\hline III & 7 & 4,0 & 10 & 37,1 & $p<0,05$ \\
\hline IV & 2 & 1,02 & 3 & 11,1 & $\mathrm{p}<0,05$ \\
\hline
\end{tabular}

Tabla 4. Complicaciones intrahospitalarias en 266 pacientes adultos con abdomen agudo

\begin{tabular}{|lrrrrr|}
\hline Tipo & \multicolumn{2}{c}{ Grupo A } & \multicolumn{2}{c|}{ Grupo B } & \\
& $\mathrm{n}$ & $\%$ & $\mathrm{n}$ & $\%$ & \\
\hline Infección herida & 13 & 5,9 & 4 & 8,9 & $\mathrm{p}$ NS \\
Infección extra-abdominal & 6 & 2,7 & 8 & 17,8 & $\mathrm{p}<0,05$ \\
Sepsis & 5 & 2,3 & 6 & 13,3 & $\mathrm{p}<0,05$ \\
Insuficiencia renal & 3 & 1,4 & 6 & 13,3 & $\mathrm{p}<0,05$ \\
Cardiovascular & 0 & - & 6 & 13,3 & $\mathrm{p}<0,05$ \\
Otras & 12 & 5,5 & 4 & 8,9 & $\mathrm{p}$ NS \\
\hline
\end{tabular}


un caso de colangitis aguda también ASA III que se trató endoscópicamente, y el tercero a un paciente de 85 años, ASA IV, con una necrosis intestinal por trombosis mesentérica.

El período total de hospitalización varió entre 1 y 65 días para el grupo $\mathrm{A}$, con un promedio de $5,4 \pm 7,4$ días, significativamente inferior al del grupo B (12,4+10,9 días). Destaca que del grupo A sólo 18,6\% permaneció hospitalizado más de una semana, a diferencia de 55,6\% del grupo B (Tabla 6).

\section{DisCUSIÓN}

El envejecimiento de la población chilena lleva a que, cada vez con mayor frecuencia, debamos enfrentar a pacientes adultos mayores en consultas médicas de urgencia. Se estima que a partir del año 2035, la población mayor de 65 años va a superar en número a la de jóvenes ${ }^{9}$, de ahí la preocupación reflejada en este estudio.

Durante un período de seis meses ingresaron a nuestro Hospital 45 pacientes mayores de 65 años, catalogados clínicamente como portadores de un abdomen agudo. En este grupo, de edad promedio 75,7 años, dos tercios presentaba patología asociada, y en más de la mitad de los casos ésta era múltiple. Ello ha sido observado por otros autores, en proporción similar ${ }^{11}$.

El diagnóstico final de estos pacientes sufrió variaciones respecto del planteado al ingreso en similar proporción en ambos grupos, independiente de la edad; ello ocurrió en 15,6\% del grupo B y 18,1\% de los menores de 65 años. Esta observación es similar a lo notificado por van Geloven $^{12}$, que señaló una variación de $20 \%$, apoyado en exámenes, cirugía o necropsia.

La causa del abdomen agudo en $71,4 \%$ de los casos de la población menor de 65 años responde sólo a dos etiologías: apendicular $(47,9 \%)$ y biliopancreática (23,5\%). Para los pacientes de más de 65 años los diagnósticos de AA cambian significativamente. El $71 \%$ de los casos está dado por cuatro causas: patología bilio-pancreática (31,1\%), obstrucción intestinal $(17,7 \%)$, hernia complicada de la pared abdominal (13,3\%) y enfermedad ulcerosa péptica (8,9\%). La apendicitis aguda fue significativamente menos frecuente en este grupo

Tabla 5. Evolución de 266 pacientes con abdomen agudo

\begin{tabular}{|c|c|c|c|c|c|}
\hline \multirow[t]{2}{*}{ Tipo } & \multicolumn{2}{|c|}{ Grupo A } & \multicolumn{2}{|c|}{ Grupo B } & \\
\hline & $\mathrm{n}$ & $\%$ & $\mathrm{n}$ & $\%$ & \\
\hline Ingreso UCI & 6 & 2,7 & 11 & 24,4 & $p<0,05$ \\
\hline Días $(\mathrm{x} \pm \mathrm{DS})$ & \multicolumn{2}{|c|}{$9,6 \pm 2,0$} & \multicolumn{2}{|c|}{$6,0 \pm 4,4$} & \\
\hline Ventilación mecánica & 3 & 1,4 & 4 & 8,9 & $p<0,05$ \\
\hline Días ( $\mathrm{x} \pm \mathrm{DS})$ & \multicolumn{2}{|c|}{$9,6 \pm 7,8$} & \multicolumn{2}{|c|}{$5,0 \pm 2,2$} & \\
\hline Complicaciones & 27 & 12,2 & 14 & 31,1 & $p<0,05$ \\
\hline Mortalidad global & 1 & 0,5 & 3 & 6,7 & $p<0,05$ \\
\hline Mortalidad quirúrgica & 1 & 0,6 & 3 & 11,1 & $\mathrm{p}<0,05$ \\
\hline
\end{tabular}

Tabla 6. Tiempo de hospitalización en 266 pacientes ingresados por abdomen agudo

\begin{tabular}{|c|c|c|c|c|c|}
\hline \multirow[t]{2}{*}{ Días } & \multicolumn{2}{|c|}{ Grupo A } & \multicolumn{2}{|c|}{ Grupo B } & \\
\hline & $\mathrm{n}$ & $\%$ & $\mathrm{n}$ & $\%$ & \\
\hline $0-3$ & 124 & 56,1 & 10 & 22,2 & $p<0,05$ \\
\hline $4-7$ & 56 & 25,3 & 10 & 22,2 & $\mathrm{p}$ NS \\
\hline & 41 & 18,6 & 25 & 55,6 & $p<0,05$ \\
\hline Total $(\mathrm{x} \pm \mathrm{DS})$ & \multicolumn{2}{|c|}{$5,4 \pm 7,4$} & $12,4+10,9$ & (1-45) & $\mathrm{p}<0,05$ \\
\hline
\end{tabular}


etario y sólo representó el 6,7\% de los cuadros. Igualmente son menos frecuentes los cuadros de origen ginecológico y los de dolor abdominal no específico como causa de hospitalización. La prevalencia de los cuatro diagnósticos más frecuentemente encontrados en esta serie de AA del adulto mayor es concordante con lo señalado por otros autores ${ }^{2,4-6}$. Los diagnósticos planteados condujeron a un tratamiento quirúrgico en $64,4 \%$ de los casos, incluyendo dos pacientes $(4,4 \%)$ que se resolvieron exclusivamente por medio de cirugía endoscópica. En una serie de la Clínica Mayo ${ }^{13}$, sólo $42 \%$ de los pacientes requirió cirugía. Otra serie ${ }^{12}$ señala que, de 132 pacientes mayores de 80 años ingresados por un AA, el 27\% fue operado. En la presente serie, para la población menor de 65 años, y concordante con los diagnósticos causales, se requirió cirugía más frecuentemente $(81,9 \%)$.

Ahora bien, la mortalidad global en nuestra serie fue significativamente mayor para el grupo de más edad, en comparación con el de menos de 65 años, alcanzando 6,7\% y 0,8\%, respectivamente, cifras que aun elevadas, se comparan favorablemente con 11,8\% de González ${ }^{14}, 13 \%$ reportado por Miettinen 6 , $17 \%$ de Paajanen ${ }^{15}$ y $22 \%$ de Kettunen ${ }^{2}$. Una mortalidad similar es señalada por una serie japonesa reciente ${ }^{7}$.

En la serie publicada por van Geloven ${ }^{12}$, la mortalidad para todos los pacientes mayores de 80 años ingresados al hospital por un AA, fue de $17 \%$, valor que se elevó al $34 \%$ para el grupo operado. En esta serie, la mortalidad para el grupo quirúrgico fue de $11,1 \%$. Datos similares han sido reportados por Wilkins ${ }^{16}$. Esta elevada mortalidad en el paciente mayor es multifactorial. Se ha reconocido que la edad por sí sola no es un factor determinante, y la mortalidad está influida por la oportunidad de la cirugía, la duración de la misma y muy especialmente por las patologías asociadas y el menoscabo funcional que de ellas resulte. Por ese motivo es que el grado de clasificación ASA es un excelente predictor de mortalidad $2,11,17-19$. En nuestras observaciones casi la mitad de los pacientes añosos era ASA III o IV, y los tres casos fatales se encontraban en esas categorías.

Dentro de los diagnósticos más relevantes como causa de AA en el adulto mayor señalamos que destacan los relacionados con la patología bilio-pancreática y de la pared abdominal. Ello es de mucha importancia porque en ambos casos se trata de condiciones que generalmente son conocidas y acompañan al paciente hasta edades avanzadas, donde su tratamiento de urgencia se asocia a una más elevada morbilidad y mortalidad que la observada en población de edad menor o de edad similar pero en condiciones de resolución electiva. Keller ${ }^{4}$ comparó mortalidad en cirugía abdominal de urgencia y electiva en pacientes sobre 70 años de edad, y ésta fue de 1,9\% para los casos electivos y de $20 \%$ para la cirugía de urgencia.

$\mathrm{Al}$ analizar diagnósticos específicos, la diferencia a favor de la cirugía electiva es siempre marcada. En una serie de patología biliar litiásica en pacientes mayores de 75 años ${ }^{11}$, la morbimortalidad en cirugía de urgencia fue de $19 \%$ y $1,7 \%$, muy superior al $10 \%$ y $0,3 \%$, respectivamente, que se encuentra en cirugía electiva. Datos concordantes han sido señalados en otras series $8,14,20$, en que la morbilidad y mortalidad es generalmente cinco a seis veces mayor en cirugía de urgencia que en cirugía electiva ${ }^{5}$. Una publicación reciente ${ }^{21}$ señala una mortalidad de $10 \%$ para la colecistectomía de urgencia en pacientes añosos de bajo riesgo, elevándose a $46 \%$ si existen significativas comorbilidades. En estos casos se ha recomendado la colecistostomía percutánea, que con un muy bajo riesgo es exitosa en más de $90 \%$ de los casos.

Las urgencias derivadas de patología ulcerosa péptica son frecuentes en la población geriátrica y se estima que entre $5 \%$ y $10 \%$ se complica de perforación ${ }^{22}$. En parte, esto puede estar condicionado por la frecuente utilización de drogas antiinflamatorias no-esteroidales en este grupo de pacientes 23,24 .

La apendicitis aguda, si bien no se cuenta entre las causas más frecuentes de AA en el adulto mayor $5,6,25,26$, es una patología que se asocia a una elevada morbilidad y mortalidad. En la serie de Paajanen ${ }^{25}$, la mortalidad fue de $21 \%$, con casi la mitad de los casos complicados de perforación y peritonitis. De ahí la necesidad de realizar oportunamente el diagnóstico. Conociendo ${ }^{23,27}$ que la sola evaluación clínica en estos casos es a menudo insuficiente para excluir el diagnóstico, debe realizarse un esfuerzo especial en la población mayor para descartar su presencia mediante exámenes de imágenes.

Considerando, entonces, el diagnóstico de apendicitis y patología biliar, parece aconsejable 
una exploración abdominal con ultrasonido en el adulto mayor en que se configura un abdomen agudo y no existe patología herniaria complicada, antes de decidir un manejo ambulatorio. Otros apoyan, incluso, la utilización liberal de la tomografía axial computada ${ }^{22,28}$. La radiografía simple de abdomen tiene, en general, una muy baja sensibilidad en patología abdominal aguda e incluso sólo llega a 49\% de los casos de obstrucción intestinal ${ }^{28}$.

Este grupo de pacientes de más de 65 años requirió más frecuentemente admisión en Unidad de Cuidados Intensivos y conexión a ventilación mecánica que el grupo de los pacientes más jóvenes, $24,4 \%$ y $2,7 \%$ vs $8,9 \%$ y $1,4 \%$, respectivamente. En la serie de Kettunen ${ }^{2}, 42 \%$ requirió de manejo en Cuidados Intensivos.

El tiempo de hospitalización fue significativamente más prolongado en el grupo de mayor edad, alcanzando 12,4 días en promedio, comparable con lo señalado por otros estudios: 12 días por Kettunen ${ }^{2}, 12$ días por Le $\mathrm{Neel}^{29}$ y 12,5 en la

\section{REFERENCIAS}

1. FenYo G. Acute abdominal disease in the elderly: Experience from two series in Stockholm. Am J Surg 1982; 143: 751-4.

2. Kettunen J, Pajanen H, Kostiainen S. Emergency abdominal surgery in the elderly. Hepatogastroenterology 1995; 42: 106-8.

3. González R, González JA. Cirugía general en el anciano. Consideraciones básicas. Rev Chil Cir 2001; 53: 7-19.

4. Keler SM, Markovitz LJ, Wilder JR, Aufses AH Jr. Emergency and elective surgery in patients over age 70. Am Surg 1987; 53: 636-40.

5. Altamirano C, Catán F, Toledo G, Ormazábal J, Lagos C, Bonacic M et al. Cirugía abdominal de urgencia en el adulto mayor. Rev Chil Cir 2002; 54: 654-7.

6. Miettinen P, Pasanen P, Salonen A, Latineen J, Alhava E. The outcome of elderly patients after operation for acute abdomen. Ann Chir Gynaecol 1996; 85: 11-5.

7. Nishida $K$, Okinaga $K$, Miyazana $Y$, Suzuki $K$, Tanaka M, Hatano M et al. Emergency abdominal surgery in patients aged 80 years and older. Surg Today 2000; 30: 22-7. serie de Miettinen ${ }^{6}$. Más de la mitad de los pacientes permaneció hospitalizado por más de una semana, a diferencia de lo que ocurrió con el grupo de menor edad, en que sobre $80 \%$ fue dado de alta dentro de los siete días.

En suma, la población de más de 65 años que ingresa por un cuadro de abdomen agudo presenta las siguientes características: 1. Es frecuentemente portadora de patologías asociadas que determinan una categoría ASA de riesgo alta. 2. Habitualmente, el diagnóstico responde a cuatro condiciones: patología bilio-pancreática, obstrucción intestinal, hernia de la pared abdominal complicada y enfermedad ulcerosa péptica. En el reconocimiento de ellas puede ser muy útil un examen abdominal por ultrasonido. Estos pacientes demandan una atención más compleja y tienen una elevada morbimortalidad ${ }^{30,31}$. Su control parece depender de la evaluación cuidadosa del cuadro agudo y probablemente, de la corrección electiva de las patologías abdominales conocidas.

8. Reiss R, Deutsch AA, Nudelman I, Gutman $H$. Multifactorial analysis of prognostic factors in emergency abdominal surgery in patients above 80 years. Analysis of 154 consecutive cases. Int Surg 1989; 74: 93-6.

9. Instituto Nacional de Estadísticas (INE). Enfoques estadísticos $\mathrm{N}^{\circ} 8$. Agosto 2000. Santiago de Chile.

10. Owens WD, Felts Ja, Sittznagel EL ASA physical status classification: A study of consistency of ratings. Anesthesiology 1978; 49: 239-43.

11. Espinoza R, López F, Guzmán S, Arroyo C, Ibáñez L, Guzmán S ET AL. Patología biliar litiásica en mayores de 75 años. Rev Chil Cir 1997; 49: 153-6.

12. Van Geloven AA, Biesheuvel TH, Luitse JS, Hoitsma HF, ОвеRTOP H. Hospital admissions of patients aged over 80 with acute abdominal complaints. Eur J Surg 2000; 166: 866-71.

13. Buglosi TF, Meioy TD, Vukov LF. Acute abdominal pain in the elderly. Ann Emerg Med 1990; 19: 1383-6.

14. González JJ, Sanz L, Grana JL, Bermejo G, Navarrete F, Martínez E. Biliary lithiasis in the elderly patient: morbidity and mortality due to biliary surgery. Hepatogastroenterology 1997; 44: 1565-8.

15. Paajanen $H$, Jahkolia M, Oksanen $H$, Nordback I. Acute pancreatitis in patients over 80 years. Eur J Surg 1996; 471-5. 
16. Wilkins GA, Vareia-Rueda CE, Biasco Y, González JM. Morbidity and mortality in patients presenting with acute abdominal pain. Rev Gastroenterol Mex 1989; 54: 223-9.

17. Arenal JJ, Bengoechea-BeEby M. Mortality associated with emergency abdominal surgery in the elderly. Can J Surg 2003; 46: 111-6.

18. EL-Haddawi F, Abu-Zidan FM, Jones W. Factors affecting surgical outcome in the elderly at Auckland Hospital. ANZ J Surg 2002; 72: 537-41.

19. HAL JC, HAL JL ASA status and age predict adverse events after abdominal surgery. J Qual Clin Pract 1996; 16: 103-8.

20. Lai EC, Tam PC, Paterson IA, Ng MM, Fan ST, Choi TK ET AL. Emergency surgery for severe acute cholangitis. The high-risk patients. Ann Surg 1990; 211: 55-9.

21. Byrne MF, Suhocki P, Mitchell RM, Pappas TN, Stiffler HL, Jowelus et al. Percutaneous cholecystostomy in patients with acute cholecystitis: Experience of 45 patients at a US referral center. J Am Coll Surg 2003; 197: 206-11.

22. Dang C, Aguitera P, Dang A, Saifm L Acute abdominal pain. Four classifications an guide assessment and management. Geriatrics 2002; 57: 30-2, 35-6, 41-2.

23. Espinoza R, Rodríguez A. Traumatic and nontraumatic perforation of hollow viscera. Surg Clin North Am 1997; 77: 1291-304.
24. Coleman JA, Denham JM. Perforation of the peptic ulceration in the elderly. Age Ageing 1980; 9: 25761.

25. Paajanen H, Kettunen J, Kostiainen S. Emengency appendectomies in patients over 80 years. Am Surg 1994; 60: 950-3.

26. Alvarez R, Bustos A, Torres O, Cancino A. Apendicitis aguda en mayores de 70 años. Rev Chil Cir 2002; 54: 345-9.

27. Espinoza R, Ohmke J, García-Huidobro I, Guzmán S, Azócar M. Apendicectomía negativa: Experiencia en un hospital universitario. Rev Méd Chile 1998; 126: 75-80.

28. Ahn SH, Mayo-Smith WW, Murphy BL, Reinert BE, CRONAN JJ. Acute nontraumatic abdominal pain in adult patient: abdominal radiography compared with CT evaluation. Radiology 2002; 225: 159-64.

29. Le Neel JC, Guiberteau B, Borde L, Kohen M, Eloufir M, Burgos-Suare B. Management of patients over 75 years of age with digestive or abdominal disease. A series of 660 cases. Chirurgie 1993-94; 119: 143-7.

30. Kim JP, Kim SJ, Lee JH, Kim SW, Chог MG, Yu HJ. Surgery in the aged in Korea. Arch Surg 1998; 133: 18-23.

31. WALSH TH. Audit of outcome of major surgery in the elderly. Br J Surg 1996; 83: 92-7. 